\title{
COHOMOLOGY OF ALGEBRAIC GROUPS AND INVARIANT SPLITTING OF ALGEBRAS ${ }^{1,2}$
}

\author{
BY EARL J. TAFT
}

\author{
Communicated by D. Zelinsky, August 16, 1966
}

1. Introduction. Let $A$ be an algebra, over a field $F$, assumed at first to be associative and finite-dimensional over $F$. Let $R$ be the radical of $A, C$ the center of $A$. Assume $A / R$ separable, so that $A$ possesses maximal separable subalgebras (Wedderburn factors) $S$ for which $A=S+R, S \cap R=0$. Let $G$ be a group of automorphisms and antiautomorphisms of $A$. We will discuss the existence and uniqueness of $G$-invariant Wedderburn factors in terms of various cohomology groups of $G$. In general, the cohomology is that of abstract groups. However, the conditions given will be compatible with taking the algebraic hull of $G$ (in the Zariski topology with respect to $F$ ), so that we can assume $G$ is an algebraic group and the cohomology is rational. We will outline here how the cohomology enters. Details will appear elsewhere. See [3], [4], [5] for a general background of the question.

2. Existence. We first assume $R^{2}=0$. Let $S$ be any maximal separable subalgebra. If $g \in G$, then $S g$ is another maximal separable subalgebra, so by the Malcev theorem, $S g=S C_{1-z(\theta)}$, where $C_{w}$ is conjugation by $w . z(g)$ is in $R$, but is uniquely determined modulo $R \cap C$, so that we consider $z$ as a function from $G$ to the vector space $R / R \cap C$. We consider $R / R \cap C$ as a $G$-module in the obvious way, except that the antiautomorphisms in $G$ act via their negatives. Then a technical calculation will show that $z \in Z^{1}(G, R / R \cap C)$, i.e., $z(g h)=z(g) \cdot h$ $+z(h)$. Hence if $H^{1}(G, R / R \cap C)=0$, there is an $x$ in $R$ such that $z(g)=x-x \cdot g+R \cap C$. A technical calculation will then show that $S C_{1-x}$ is a $G$-invariant maximal separable subalgebra.

Now we consider the general case $R^{2} \neq 0$. The action of $G$ on all modules will be the obvious ones, except that the antiautomorphisms in $G$ will act via their negatives. We consider $A / R^{2}$. The condition for the case $R^{2}=0$ above now becomes $H^{1}\left(G, R /\left\{x \in R \mid[A, x] \subseteq R^{2}\right\}\right)$ $=0$ where $[A, x]=\{[a, x]=a x-x a \mid a \in A\}$. If this holds, then $A=S_{1}+R, S_{1}$ a $G$-invariant subalgebra, $S_{1} \cap R \subseteq R^{2} . S_{1}$ has radical $R^{2}$, and we next consider $S_{1} / R^{4}$. The condition now is

${ }^{1}$ This research was supported by the National Science Foundation Grant GP-4157.

2 Presented to the Society January 27, 1966. 
$H^{1}\left(G, R^{2} /\left\{x \in R^{2} \mid\left[S_{1}, x\right] \subseteq R^{4}\right\}\right)=0$. This yields $A=S_{2}+R, S_{2}$ a $G$ invariant subalgebra, $S_{2} \cap R \subseteq R^{4}$. Let $R^{2^{n}} \neq 0, R^{2^{n+1}}=0$. Then the conditions become $H^{1}\left(G, R^{2^{i}} /\left\{x \in R^{2^{i}} \mid\left[x, S_{i}\right] \subseteq R^{2^{i+1}}\right\}\right)$ for $i=0,1$, $\cdots, n$, where $S_{0}=A, S_{1}, \cdots, S_{n}$ are $G$-invariant subalgebras as indicated. The next step yields $S_{n+1}$ as a $G$-invariant maximal separable subalgebra.

3. Applications. All the modules considered are rational modules for the algebraic hull of $G$, and the cocycles are rational functions. Hence we may assume $G$ is an algebraic group. If $G$ is reductive, then the rational cohomology $H^{1}(G, M)=0$ for $M$ a rational $G$-module. This follows from an argument in [1] as follows: Let $W=F \oplus M$, $f \in Z^{1}(G, M)$. Let $G$ act on $W$ by $(a, m) g=(a, m g+f(g))$. $W$ is completely reducible since $G$ acts rationally on it. Let $C$ be a $G$-complement to $M$ in $W . C$ has a unique element $(1, x), x$ in $M$. Applying $g \in G$ yields $f(g)=x-x g$. This argument shows that $A$ possesses $G$ invariant maximal separable subalgebras if the algebraic hull of $G$ is a reductive algebraic group. In particular, it holds if $F$ has characteristic zero and $G$ is completely reducible (see [2]).

The cohomology conditions are well-known if $G$ is a finite group of order not divisible by the characteristic of $F$.

Note that $\left\{x \in R^{2^{i}} \mid\left[x, S_{i}\right] \subseteq R^{2^{i+1}}\right\}$ is a Lie ideal in $S_{i}$. This indicates that similar results hold for Lie algebras over fields of characteristic zero.

By inducting on the degree of nilpotency of $R$, rather than on the dimension of $A$, we note that the cohomology conditions (for abstract groups) will suffice for infinite-dimensional algebras (with nilpotent radicals), provided the algebras involved possess Wedderburn principal decompositions which satisfy the Malcev theorem.

See also [5] for additional results concerning existence.

4. Uniqueness. Here the aim is to prove that any two $G$-invariant maximal separable subalgebras $S$ and $T$ are $G$-orthogonally conjugate (see [4] for definitions). Again, $G$ will act naturally on the modules which arise, except that the antiautomorphisms act via their negatives. Also let characteristic $F \neq 2$.

First let $R^{2}=0, z$ in $R$ so that $S C_{1-z}=T$. A technical calculation yields $z-z \cdot g$ in $R \cap C$, and $f(g)=z-z \cdot g$ satisfies $f$ in $Z^{1}(G, R \cap C)$. If $H^{1}(G, R \cap C)=0$, then there is an $x$ in $R \cap C$ so $z-z \cdot g=x-x \cdot g$. Then $z-x$ is $G$-skew (called $G$-symmetric in [4]) so $1-z+x$ is $G$ orthogonal, and $S C_{1-z+x}=T$.

Now consider the general case. The cohomology condition for $A / R^{2}$ becomes $H^{1}\left(G,\left\{x \in R \mid[T, x] \subseteq R^{2}\right\} / R^{2}\right)=0$. Then this yields 
$z_{1}$ in $R$ so that $z_{1}+R^{2}$ is $G$-skew in $R / R^{2}$ and $S C_{1-z_{1}}+R^{2}=T+R^{2}$. Define $f_{1}(g)=\frac{1}{2}\left(z_{1} \cdot g-z_{1}\right)$. Then $f_{1} \in Z^{1}\left(G, R^{2}\right)$. If $H^{1}\left(G, R^{2}\right)=0$, we get an $x_{1}$ in $R^{2}$ so $f_{1}(g)=x_{1}-x_{1} \cdot g$. Let $y_{1}=-x_{1}-z_{1} / 2$. Then $y_{1}$ is $G-$ skew and $y_{1}+R^{2}=-z_{1} / 2+R^{2}$. Let $u_{1}=-2 y_{1}\left(1-y_{1}\right)^{-1}$. Then $1-u_{1}$ is $G$-orthogonal and $S C_{1-u_{1}}+R^{2}=T+R^{2}$.

Repeating these arguments, two sets of conditions emerge. They are (1) $H^{1}\left(G,\left\{x \in R^{i^{i}} \mid[T, x] \subseteq R^{2^{i+1}}\right\} / R^{2^{i+1}}\right)=0, i=0,1, \cdots, n$, and (2) $H^{1}\left(G, R^{2^{i}}\right)=0, i=1, \cdots, n$. The first set yields $G$-skew cosets. The second set enables one to lift out suitable $G$-skew elements out of which are built $G$-orthogonal elements using a Cayley transform. Finally $\left(1-u_{1}\right)\left(1-u_{2}\right) \cdots\left(1-u_{n}\right)\left(1-u_{n+1}\right)$ conjugates $S$ into $T$.

The same general remarks in $\$ 3$ apply here also. However, we point out that for finite-dimensional algebras the conclusion can be proved for any completely reducible group, even if the characteristic is not zero (but not two), as in [4].

\section{REFERENCES}

1. G. Hochschild, Cohomology of algebraic linear groups, Illinois J. Math. 5 (1961), 492-519.

2. G. D. Mostow, Fully reducible subgroups of algebraic groups, Amer. J. Math. 78 (1956), 200-221.

3. E. J. Taft, Invariant Wedderburn factors, Illinois J. Math. 1 (1957), 565-573.

4. - Orthogonal conjugacies in associative and Lie algebras, Trans. Amer. Math. Soc. 113 (1964), 18-29.

5. - On certain d-groups of algebra automorphisms and antiautomorphisms, J. Algebra 3 (1966), 115-121.

Rutgers, The State University 